\title{
Dispositivos intraorales utilizados para el tratamiento de la apnea obstructiva del sueño en pacientes edéntulos. Revisión de la literatura y bases de patentes
}

Intraoral Devices for OSA Treatment in Edentulous Patients. Literature and Patents Datebase Review

Roneith Alexandra Galdón Torres

Pontificia Universidad Javeriana, Colombia

Vanessa Peña Peti ${ }^{2}$

Pontificia Universidad Javeriana, Colombia

Daniel Zuluaga ${ }^{3}$

Pontificia Universidad Javeriana, Colombia

Ángela Suárez Castillo 4

Pontificia Universidad Javeriana, Colombia

Laura Catalina Lara Hernández ${ }^{5}$

Pontificia Universidad Javeriana, Colombia

Liliana Margarita Otero Mendoza ${ }^{6}$

Pontificia Universidad Javeriana, Colombia

Patricia Hidalgo Martínez ${ }^{7}$

Pontificia Universidad Javeriana, Colombia

1 Odontóloga. Residente del Posgrado de Ortodoncia, Facultad de Odontología, Pontificia Universidad Javeriana, Bogotá, Colombia.

2 Odontóloga. Residente del Posgrado de Ortodoncia, Facultad de Odontología, Pontificia Universidad Javeriana, Bogotá, Colombia.

3 Estudiante de Semillero, Facultad de Odontología, Pontificia Universidad Javeriana, Bogotá, Colombia.

4 Odontóloga. Profesora de la Facultad de Odontología, Pontificia Universidad Javeriana, Bogotá, Colombia.

5 Odontóloga. Profesora de la Facultad de Odontología, Pontificia Universidad Javeriana, Bogotá, Colombia.

6 Odontóloga. Profesora de la Facultad de Odontología, Pontificia Universidad Javeriana, Bogotá, Colombia.

7 Médica. Profesora de la Facultad de Medicina, Pontificia Universidad Javeriana y Clínica del Sueño, Hospital Universitario San Ignacio, Bogotá, Colombia.

Cómo citar: Galdón Torres RA, Peña Petit V, Zuluaga D, Suárez Castillo Á, Lara Hernández LC, Otero Mendoza LM, Hidalgo Martínez P. Dispositivos intraorales utilizados para el tratamiento de la apnea obstructiva del sueño en pacientes edéntulos. Revisión de la literatura y bases de patentes. Univ Med 2017;58(1):1-6. $\quad$ https://doi.org/10.11144/ Javeriana.umed 58-1.diao

\section{RESUMEN}

Introducción: la apnea obstructiva del sueño (AOS) se considera un problema de salud pública que afecta en gran medida a hombres y mujeres entre 50 y 70 años de edad, donde en ocasiones son, además, pacientes edéntulos, lo cual dificulta su tratamiento. Objetivo: identificar los aparatos intraorales existentes para tratar la AOS leve y moderada en pacientes edéntulos. Metodología: se buscó literatura sobre el tema en bases de datos y de patentes. Resultados: se encontraron cinco artículos y dos patentes. El objetivo terapéutico de todos los dispositivos es aumentar la vía aérea superior entre la base de la lengua y la pared posterior de la faringe, mediante el avance mandibular. Dentro de las principales desventajas que presentan estos dispositivos están falta de retención, dificultad para mantener la dimensión vertical, problemas musculares y trastornos en la articulación temporomandibular. Conclusión: existen pocos dispositivos intraorales efectivos en la literatura para el tratamiento de la AOS en pacientes edéntulos. Esto hace necesario diseñar futuras investigaciones en esta área.

Palabras clave

apnea obstructiva del sueño; tratamiento; edéntulos. 


\begin{abstract}
Introduction: The Obstructive Sleep Apnea (OSA) is considered a problem of World Public Health that greatly affects men and women between 50 and 70 years old, where some of them are edentulous patients which treatment difficulty. Objective: To identify the existing devices for mild and moderate OSA treatment in edentulous patients. Method: A comprehensive review was conducted in the literature and patent databases. Results: 5 articles and 2 patents were found. The main therapy objective in all devices is to increase the upper airway space between the tongue base and the posterior pharyngeal wall trough mandibular advancement. Several problems with use of these devices include lack retention, difficult for maintain the vertical dimension, muscular discomfort and Temporomandibular Disorders. Conclusion: There are limited effective intraoral devices in literature for OSA treatment in edentulous patients. It is necessary to design future investigations in this area.

Keywords

obstructive sleep apnea; treatment; edentulous.
\end{abstract}

\section{Introducción}

La apnea obstructiva del sueño (AOS) es una condición que se caracteriza por episodios frecuentes de obstrucción de la vía aérea superior (VAS) durante el sueño, lo que resulta en la reducción de la saturación de la cantidad de oxígeno en la sangre y, por ende, despertares transitorios [1]. Generalmente, la obstrucción de la VAS ocurre durante el intento de inspiración, ya sea por colapso del paladar blando, de la base de la lengua contra la pared posterior de la faringe, o en ambos sitios, donde luego de al menos diez segundos sin ventilación adecuada el paciente se despierta brevemente, inhala y luego vuelve a conciliar el sueño [2]. Esta condición es de origen multifactorial, en la cual componentes como la obesidad, las anomalías craneofaciales, la edad, el género, la etnia, factores ambientales y condiciones congénitas y adquiridas pueden aumentar la predisposición al colapso de la VAS [3].

Hoy en día, la AOS se considera un problema de salud pública, porque puede llegar a ser una condición letal, debido a que se ha observado que la obstrucción de la VAS en cualquier segmento tiende a desencadenar hipoxemia, hipercapnia e incremento del esfuerzo ventilatorio, lo que aumenta el riesgo de desarrollar problemas cardiacos [4]. Aunado a esto, en los últimos años se ha reportado un incremento progresivo de la prevalencia de la AOS en el mundo tanto en hombres como en mujeres. En el 2012, Ayik y Akhan [5] y Correa [6], en el 2013, mencionaron que la AOS presenta una prevalencia del $30 \%$ al $80 \%$ en adultos mayores, comparada con la prevalencia de la población de adultos jóvenes, que es del 2 $\%$ en mujeres y del $4 \%$ en hombres. Por otra parte, Ha, Hirai y Tsoi, en el 2014 [7], mencionan que la prevalencia de AOS según el sexo es del $3 \%$ al $7 \%$, aproximadamente, en hombres adultos y del $2 \%$ al $5 \%$ en mujeres, lo que evidencia un aumento en la prevalencia a lo largo de los años.

En el 2013, Mehta y colegas [8], en una revisión de la literatura, encontraron que la prevalencia de la AOS en la población general es del $2 \%$ al $25 \%$. En Colombia, Escobar y Liendo, en el 2012 [9], reportaron que la prevalencia de los trastornos del sueño era del 27 \%; mientras que Ruiz y colegas, en el 2016 [10], en su estudio sobre la prevalencia de alteraciones de sueño en Colombia a diferentes altitudes, reportaron que la prevalencia global de trastornos de sueño fue del 59,6\%.

En cuanto a la edad, la literatura revela que la AOS, generalmente, está asociada a adultos mayores, quienes tienden a presentar trastorno sistémico y algún tipo de edentulismo que puede favorecer la aparición de la AOS [11]. Según el reporte del Estudio Nacional de Salud Bucal del Ministerio de Salud (ENSAB IV) 2013-2014 en Colombia, la prevalencia de pacientes edéntulos fue del 40,8 \% de la población entre 35 y 79 años de edad [12]. El edentulismo puede ser otro factor contribuyente para la aparición de la AOS, debido a que los principales cambios anatómicos y esqueléticos causados por la pérdida de los dientes naturales, como son pérdida de la dimensión vertical, reducción de la altura facial inferior y rotación de la mandíbula, a largo plazo tienden a generar en la lengua la obtención de una posición anormal, que puede contribuir con obstrucción de la vía aérea [11].

Por otra parte, se ha descrito que la relación esquelética o la presencia de ciertas anomalías craneofaciales pueden incrementar el riesgo de desarrollar AOS. Dobrowolska y colegas [13], en 
el 2013, observaron en radiografías laterales que el tamaño de las vías aéreas tiene una estrecha relación con el ángulo ANB (ángulo formado por los planos Nasion, punto A y punto B), lo cual sugiere que patrones esqueléticos clase II tendrán vías aéreas disminuidas; mientras que patrones esqueléticos clase III tienden a tener vías aéreas aumentadas. Por otra parte, Gungor y colegas [14], en el 2013, demostraron igualmente, por medio de radiografías laterales tomadas en pacientes con AOS y sujetos sanos, que las características faciales y esqueléticas, como SNA (ángulo formado por Silla-Nasion-punto A), SNB (ángulo formado por Silla-Nasion-punto B) y el ángulo del plano mandibular (GoGn-SN), eran similares en ambos grupos. Por lo tanto, no existe un patrón concluyente en cuanto a esta característica. Aunque la radiografía lateral de cráneo puede utilizarse como una herramienta complementaria para el diagnóstico de la AOS [15], comúnmente, el diagnóstico inicia a partir de una sospecha clínica derivada de síntomas como somnolencia diurna excesiva, sueño no reparador, ronquidos, entre otros [16].

Para obtener un diagnóstico pertinente, es necesario iniciar con una evaluación que incluya la historia clínica del paciente, la exploración física general y la realización de diferentes estudios e interconsultas [16,17]. El principal estudio para el diagnóstico de la AOS es el polisomnograma nocturno, considerado el patrón de referencia [18], el cual registra los índices de saturación de oxígeno en la sangre, la frecuencia cardiaca, el flujo de aire, el índice de apnea/hipoapnea (IAH), la etapa de sueño, los movimientos de tórax y abdomen, el electromiograma y el electrooculograma.

Por otra parte, el IAH determina la severidad mediante el número de eventos de apnea e hipopnea que se presentan por hora. Se considera leve cuando se presentan entre cinco y quince eventos por hora; moderado, de quince a treinta eventos por hora, y severo, con más de treinta eventos por hora [18]. Un IAH de más de cinco eventos por hora, junto con síntomas diurnos/nocturnos, como ronquidos y somnolencia diurna, cansancio, etc., se diagnóstica como AOS [19]. Un diagnóstico pertinente facilitará un plan de tratamiento acorde a las necesidades de cada paciente.

A lo largo de los años se han propuesto diferentes alternativas para el manejo $y$ tratamiento de la AOS, entre ellas se encuentran modificaciones comportamentales como: reducir el peso, evitar la privación del sueño y el consumo de tabaco, alcohol y sedantes. Por otra parte, también existen alternativas de tratamientos como la opción quirúrgica, el uso de equipos de presión positiva de la vía aérea, farmacoterapia y el uso de dispositivos intraorales, los cuales, a pesar de ser una alternativa menos invasiva, presentan ciertas limitaciones (una de las cuales es el número insuficiente de dientes presentes en los arcos dentales) [17,18].

Teniendo en cuenta lo descrito y que la AOS, además de ser un factor de riesgo para la salud que disminuye la calidad de vida y que la prevalencia de edéntulos en el mundo y en Colombia se ha incrementado en los últimos años, surge la necesidad de realizar una búsqueda de la literatura en bases de datos y de patentes sobre alternativas de dispositivos intraorales para la AOS en pacientes edéntulos.

\section{Objetivos}

Identificar los tipos de aparatología intraoral existente para pacientes edéntulos con fines comparativos.

Identificar las limitaciones de la aparatología intraoral existente.

\section{Método}

Se realizó una búsqueda de artículos publicados entre el 2005 y el 2015 en las bases de datos Pubmed, EMBASE y Cochrane, mediante el uso de las palabras clave: apnea obstructiva del sueño, tratamiento y edéntulos. Así solo se encontraron cinco artículos que cumplían con el criterio de ser aparatos intraorales para tratar la AOS en pacientes edéntulos.

Además, mediante el uso de los mismos criterios, se realizó la inteligencia tecnológica con la búsqueda de bases de datos de patentes 
en worldwide.espacenet.com, Google Patent, WIPO, USPTO y EPO, y se encontraron solo dos patentes indicadas para esta revisión de la literatura.

\section{Resultados}

Luego de realizada la búsqueda de la literatura sobre el tema y bases de patentes para pacientes edéntulos, se desarrolló una tabla comparativa con las ventajas y las limitaciones de los dispositivos intraorales existentes encontrados (tablas 1 y 2 ).

Tabla 1. Comparativo de aparatos existentes en la literatura para edéntulos

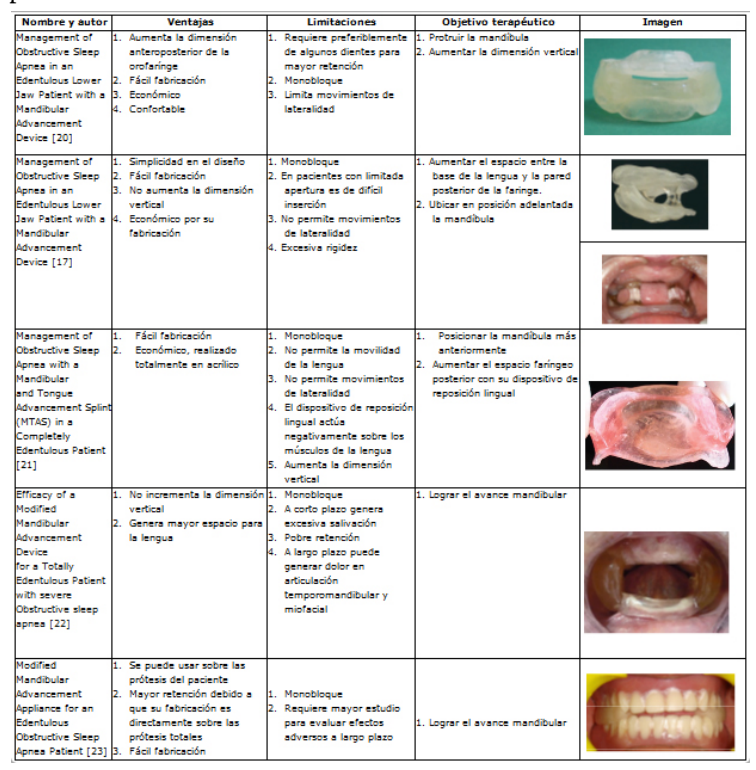

Tabla 2. Comparativo de aparatos existentes en las bases de patentes

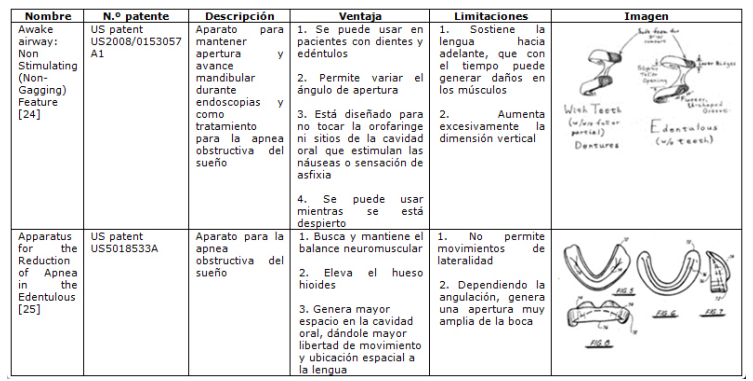

\section{Discusión}

Realizada la búsqueda en la literatura y bases de patentes e identificados los tipos de aparatología intraoral existente para el tratamiento de la AOS, se encontró que para pacientes dentados este tipo de alternativa está bien documentada; mientras que para pacientes edéntulos, aunque actualmente el tratamiento con dispositivos intraorales ha adquirido un gran auge, el número de alternativas y la evidencia sigue considerándose escasa [16]; se limita solo a estudios de casos y, en su mayoría, mediante el uso de un dispositivo de avance mandibular [26]. Esto puede atribuirse a la mayor problemática que presentan este tipo de pacientes, que es la dificultad de obtener una retención adecuada directamente por parte de los rebordes del paciente [20].

Nayar y Knox [17], en el 2005, propusieron un aparato para el tratamiento de la AOS en edéntulos totales, donde el diseño incluyó una férula convencional maxilar realizada al vacío y una férula de avance mandibular, ambas soportadas por el tejido blando del paciente y unidas entre sí por acrílico autopolimerizable. La principal limitante de este dispositivo es que se dependía de la expansión de los flancos linguales que presentaba el paciente para lograr una retención adecuada.

En el 2010, autores como Piskin y colegas [22] expusieron en su reporte de caso un aparato intraoral para edéntulos totales directamente sobre los rebordes del paciente, que involucraba avance mandibular, con el objetivo de desplazar los músculos maseteros lateralmente y así proporcionar más espacio para la lengua en pacientes con AOS grave. Por otra parte, en ese mismo año, Nelogi y colegas [23] reportaron que los aparatos intraorales, para obtener una mayor retención, necesitaban la presencia de las prótesis dentales, es decir, que el aparato intraoral debía ser utilizado sobre las prótesis del paciente. En el 2014, Keyf y colegas [20] recomendaron que la mejor opción para este tipo de pacientes son los implantes en los rebordes para utilizar aparatos implantosoportados que, además de proporcionar mayor retención, disminuyen el 
daño a los tejidos. La mayor problemática de esta opción de tratamiento se ve reflejada en el aumento del costo, debido a la necesidad de la colocación de los implantes.

La búsqueda realizada en bases de patentes y bases de datos bibliográficas permite concluir que no existe un dispositivo intraoral para el tratamiento de la AOS leve o moderada que cumpla con los requisitos necesarios para ser efectivos en cualquier paciente edéntulo, sin importar sus características intraorales individuales. Son necesarios futuros estudios que permitan diseñar un dispositivo intraoral con las condiciones para lograr efectividad en el tratamiento de la AOS y la adherencia de los pacientes a este tratamiento.

\section{Conclusiones}

Se evidenció la escasa literatura y disponibilidad de dispositivas dispositivos intraorales para pacientes edéntulos con AOS.

Al comparar los dispositivos intraorales existentes en bases de datos de patentes y reportados en la literatura se encontró que:

Ninguno de los dispositivos utiliza implantes para generar mayor retención.

Aunque todos los dispositivos logran un avance mandibular en los pacientes, este no es personalizado y no responde a las necesidades que presenta cada paciente, sino que se avanza entre el $50 \%$ y el $75 \%$ de la protrusión total del paciente.

La mayoría de los diseños encontrados son monobloques, que no le permiten al paciente realizar movimientos de lateralidad, apertura y cierre.

\section{Referencias}

1. Friedman M, editor. Sleep apnea and snoring, surgical and non-surgical therapy. New York: Saunders Elseviere; 2009.

2. Cohen R. Obstructive sleep apnea: Oral appliance therapy and severity of condition. Oral Surg Oral Med Oral Pathol Radiol Endod. 1998;85(4):388-92.
3. Abad VC. Pharmacologic therapy for obstructive sleep apnea. Sleep Medicine Clinics. 2013;8(4):527-42.

4. Páez S, Londoño N. Síndrome de apnea hipoapnea obstructiva del sueño desde el punto de vista neumológico. Acta de Otorrinolaringología y Cirugía de Cabeza y Cuello. 2011;Supl;39(3):28-35.

5. Ayik S, Akhan G. Clinical significance of obstructive sleep apnea in middle aged and elderly. European Geriatric Medicine. 2012;3(4):213-8.

6. Correa LP. Overview of oral appliance therapy for the management of obstructive sleep apnea. Sleep Med Clin. 2013;8(4):505-16.

7. Ha SCN, Hirai HW, Tsoi KKF. Comparison of positional therapy versus continuous positive airway pressure in patients with positional obstructive sleep apnea: A metaanalysis of randomized trials. Sleep Med Rev. 2014;18(1):19-24.

8. Mehta V, Vasu TS, Phillips B, Chung F. Obstructive sleep apnea and oxygen therapy: A systematic review of the literature and meta-analysis. J Clin Sleep Med. 2013;9(3):271-9.

9. Escobar-Córdoba F, Liendo C. Trastornos respiratorios del sueño y alteraciones cardiovasculares [editorial]. Rev Fac Med [internet]. 2012;60(1):1-3. Disponible en: http://www.scielo.org.co/scielo.php?script $=$ sci_arttext\&pid $=$ S0120-001120120001 $00001 \& \operatorname{lng}=$ en

10. Ruiz A, Rondón M, Hidalgo $\mathrm{P}$, et al. Prevalence of sleep complaints in Colombia at different altitudes. Sleep Sci. 2016;9(2):100-5. doi: 10.1016/ j.slsci.2016.05.008

11. Bucca C, Cicolin A, Brussino L, Arienti A, Graziano A, Erovigni F, et al. Tooth loss and obstructive sleep apnoea. Respir Res. 2006;17(7):8.

12. Ministerio de Salud de Colombia. IV Estudio Nacional de Salud Bucal (ENSAB IV): Situación en salud bucal. 2013-2014 [internet]. Disponible en: http://www.mins alud.gov.co/sites/rid/Lists/BibliotecaDigital 
/RIDE/VS/PP/ENSAB-IV-Situacion-Bucal -Actual.pdf

13. Dobrowolska M, Dunin I, Mitura I, Dąbała M. Evaluation of upper airways depth among patients with skeletal Class I and III. Folia Morphol. 2013;72(2):155-60.

14. Gungor AY, Turkkahraman H, Yilmaz HH, Yariktas M. Cephalometric comparison of obstructive sleep apnea patients and healthy controls. Eur J Dent. 2013;7(1):48-54.

15. Ryu HH, Kim CH, Cheon SM, Bae WY, Kim SH, Koo SK, et al. The usefulness of cephalometric measurement as a diagnostic tool for obstructive sleep apnea syndrome: a retrospective study. Oral Surg Oral Med Oral Pathol Oral Radiol. 2015;119:20-31.

16. Umemoto G, Yoshimura C, Aoyagi N, Toyoshima $\mathrm{H}$, Matsumoto $\mathrm{T}$, Watanabe $\mathrm{K}$, et al. Treatment outcome of the twopart semi-rigid oral appliance in obstructive sleep apnea. Oral Science International. 2012;9(2):49-54.

17. Nayar S, Knox J. Management of obstructive sleep apnea in an edentulous patient with a mandibular advancement splint: A clinical report. J Prosthet Dent. 2005;94(2):108-11.

18. Qureshi A, Ballard RD, Nelson HS. Obstructive sleep apnea. J Allergy Clin Immunol. 2003;112(4):643-51.

19. Quan SF, Howard BV, Iber C, Kiley JP, Nieto FJ, O'Connor GT, et al. The Sleep heart health study: Design, rationale, and methods. Sleep. 1997;20(12):1077-85.

20. Keyf F, Ciftci B, Firat Guven S. Management of obstructive sleep apnea in an edentulous lower jaw patient with a mandibular advancement device. Case Rep Dent. 2014;2014:436904.

21. Kurtulmus H, Cotert HS. Management of obstructive sleep apnea with a mandibular and tongue advancement splint (MTAS) in a completely edentulous patient. A clinical report. J Prosthodont. 2009;18(4):348-52.

22. Piskin B, Sentut F, Sevketbeyoglu H, Avsever H, Gunduz K, Kose M, et al. Efficacy of a modified mandibular advancement device for a totally edentulous patient with severe obstructive sleep apnea. Sleep Breath. 2010;14(1):81-5.

23. Nelogi S, Porwal A, Naveen H. Modified mandibular advancement appliance for an edentulous obstructive sleep apnea patient: A clinical report. J Prosthodont Res. 2011;55(3):179-83.

24. Baldwing $\mathrm{C}$, inventor. Awake airway: non stimulating (Non-Gagging) feature. USA patent US2008/0153057 A1. 2008 Jun 26.

25. Hawkins R, inventor. Apparatus for the reduction of apnaa in the edentulous. USA patent US patent US5018533A. 1991 May 28

26. Flanagan D. A removable snore reduction appliance for a mandibular edentulous patient. J Oral Implantol. 2009;35(5):221-4. 